\title{
DOES QUANTITATIVE CRANIAL ULTRASOUND PREDICT SEVERITY OF DISABILITY IN PREMATURE INFANTS WITH POST-HEMORRHAGIC VENTRICULAR DILATATION?
}

\author{
S. Jary ${ }^{1}$, G. Kmita ${ }^{2}$, J. Wroblewska ${ }^{3}$, E. Musialik-Swietlinska ${ }^{3}$, A. Whitelaw ${ }^{1}$ \\ ${ }^{I}$ Clinical Science, University of Bristol, Bristol, UK, ${ }^{2}$ Psychology, University of Warsaw, Warsaw, ${ }^{3}$ Neonatal \\ Intensive Care, University of Silesia, Katowice, Poland
}

Background: Infants with Post-Hemorrhagic Ventricular Dilatation (PHVD) are known to have a range of disabilities, some severe. Apart from presence of parenchymal infarction, there are few guides to prognosis.

Methods: 69 premature infants with PHVD survived until 2 years past term. Ventricular dimensions, including midline shift and Doppler blood flow velocity were measured just prior to treatment starting for PHVD. Bayley Scales of Infant Development, and components of disability at 2 years past term were examined at 2 years past term. Because almost half the infants scored below 50 on the Bayley scales, Developmental Age Equivalent was used to provide a continuous variable for all children, the Developmental Quotient (DQ).

Results: In infants with grade $3 \mathrm{IVH}$, neither maximum lateral ventricular width nor maximum ventricular cross-sectional area before treatment started, correlated with Bayley mental DQ nor motor DQ nor with inability to walk or communicate. In infants with parenchymal infarction (grade 4), the area of the lesion(s) had a negative correlation with motor DQ $(r=0.47 \mathrm{p}=0.004)$ and mental DQ $(r=0.42 \mathrm{p}=0.011)$. Bilateral parenchymal lesions had a worse prognosis than unilateral lesions. Loss of cerebral arterial diastolic velocity was associated with worse developmental outcome. Infants who eventually needed shunt surgery had larger blood clots (by area).

Discussion: Beyond the initial enlargement to $4 \mathrm{~mm}$ over the $97^{\text {th }}$ centile, further ventricular distension did not worsen developmental outcome. Measuring the location and size of parenchymal lesions and identifying pressure effects can increase precision in predicting severe motor and mental disability. 